\title{
nature
}

physics

\section{To him who waits}

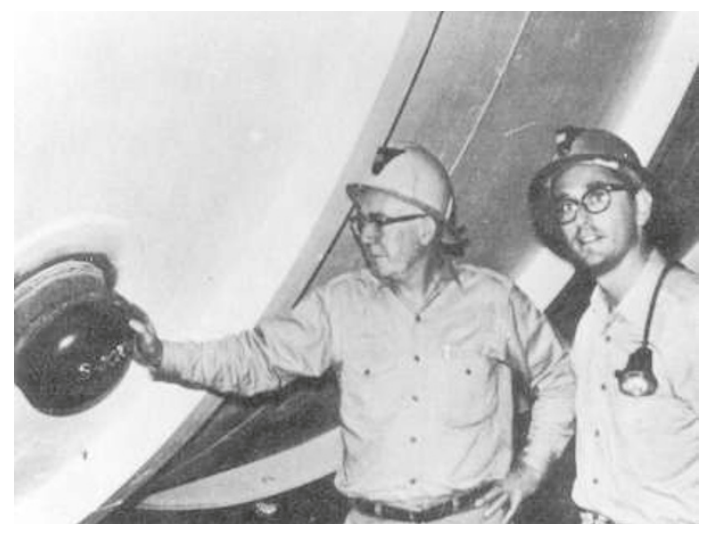

RAY DAVIS AND JOHN BAHCALL, IN THE HOMESTAKE MINE IN THE 1960 .

REFERENCES

1. Cowan, C. L. Jr et al. Science 124, 103-104 (1956).

2. Pontecorvo, B. Chalk River Laboratory Report PD-205 (1946).

3. http://nobelprize.org/physics/ laureates/2002/davis-lecture.pdf

4. Davis, R. Jr Phys. Rev. 97, 766-769 (1955).

5. Davis, R. Jr Phys. Rev. Lett. 12, 303-305 (1964).

6. Bahcall, J. N. Phys. Rev. Lett. 12, 300-302 (1964)

7. Davis, R. Jr, Harmer, D. S. \& Hoffman, K. C. Phys. Rev. Lett. 20, 1205-1209 (1968)

8. Bahcall, J. N., Bahcall, N. \& Shaviv, G. Phys. Rev. Lett. 20 1209-1212 (1968)

9. Gribov, V. \& Pontecorvo, B. Phys. Lett. B 28, 493-496 (1969).
It is 50 years since Frederick Reines and Clyde Cowan detected the neutrino ${ }^{1}$, directly and definitively. Wolfgang Pauli had postulated the existence of such a particle in 1930. On 15 June 1956, having been notified by telegram of its discovery, Pauli wrote to Reines and Cowan, "Thanks for the message. Everything comes to him who knows how to wait."

Ray Davis also knew how to wait. Davis, who died on 31 May this year, was the pioneer of experiments with solar neutrinos. With John Bahcall, who died last year, they were the originators of the 'solar neutrino problem' - a mystery that took more than 30 years to solve.

Davis graduated from Yale in 1942 with a PhD in physical chemistry. In the postwar years, he joined the newly created Brookhaven National Laboratory and began work on the recoil of nuclei following neutrino emission. By the early 1950s, he was following up the suggestion by Bruno Pontecorvo ${ }^{2}$ that neutrinos could be detected through their capture by chlorine nuclei, forming argon nuclei that could be removed and counted. "No one else appeared interested", said Davis $3^{3}$; "it seemed a natural and timely experiment for me to work on." But his detection attempt, using a 3,800-litre tank of carbon tetrachloride at one of the Savannah River reactors, seemingly failed. In fact, fission reactors are sources of antineutrinos, not neutrinos, and chlorine capture only works for neutrinos: Davis had found the first proof that neutrinos and antineutrinos are not the same ${ }^{4}$.

Pontecorvo had also mooted that the chlorine-argon detection method could be used for solar neutrinos, and by the late 1950s developments in modelling the reactions that power the Sun suggested that its neutrino flux might be detectable. Davis's 3,800-litre tank was, however, drawing a blank. But flaws were found in some of the theoretical assumptions, other nuclear reaction rates in the process were pinned down - and the situation was worse than ever. The flux of solar neutrinos seemed unmeasurable even with the largest conceivable detector.

Nevertheless, in 1964 Davis published a proposal for a new solar neutrino detector ${ }^{5}$. In a companion paper, Bahcall reported the theoretical breakthrough that would make it feasible: the rate at which chlorine nuclei capture the neutrinos from boron-8 produced in the Sun was in fact 20 times as high as previously calculated, owing to the existence of a 'superallowed' transition from the ground state in chlorine-37 to the 5-MeV 'isotopic analogue state' in argon-37. Davis got the go-ahead to build a 378,000-litre detector in the Homestake gold mine, South Dakota.

The experiment at Homestake began in 1967, but the results from what Davis called "our first good run" suggested that the solar neutrino flux was much lower than expected. Already concerned, Davis wrote jokingly to Willy Fowler, "We are ready now, turn on the Sun." When the data were formally published7 in 1968, with Bahcall's latest numbers 8 in Physical Review Letters, the pattern was set: the observations from Homestake would never muster more than a third of the predicted flux of neutrinos from the Sun. The solar neutrino problem was born.

Davis was a careful, dedicated experimenter. He checked, refined, and checked again every aspect of the process, hammering down the background and confirming the neutrino deficit. He continued to collect data at Homestake for 30 years, under the auspices of Penn State University following his retirement from Brookhaven in 1984, until the mine closed in the late 1990s. Despite a matching effort, led by Bahcall, to refine the theory, the data remained stubbornly below the prediction; the solar neutrino problem wouldn't go away.

Then in 1989 came the first confirmation of Davis's result, from the Kamiokande detector in Japan and supported by data from two other detectors, SAGE in Russia and GALLEX in Italy. But within two years, there were data from helioseismological studies that verified the theory. They couldn't both be right - but, in fact, they were. The deadlock was broken in 2001, by data from the Sudbury Neutrino Observatory (SNO), in the Inco nickel mine in Ontario. SNO confirmed the Homestake deficit of electron neutrinos, one of the three types of neutrino. But, combined with the data from Kamiokande, the results indicated that the number of neutrinos of all types emanating from the Sun matched Bahcall's model: the neutrinos just seemed to change type en route. At last, a 1969 postulation ${ }^{9}$ by Pontecorvo (him again) and Vladimir Gribov was in the spotlight: neutrinos could oscillate between their three types, electron, muon and tau; the standard model of particle physics needed updating.

Following the SNO announcement, John Bahcall was quoted in The New York Times saying, "I feel like dancing I'm so happy." Nearly four decades after their first foray into the Homestake mine, the problem thrown up by the pioneering efforts of Davis and Bahcall was solved. In 2002, Davis was a deserving recipient of the Nobel Prize: it is a shame his long-time friend and collaborator didn't share it with him. 